\title{
Monolithic microcavity with carbon nanotubes as active material
}

\author{
D. Legrand, ${ }^{1}$ C. Roquelet,${ }^{1}$ G. Lanty, ${ }^{1}$ Ph. Roussignol, ${ }^{2}$ X. Lafosse,${ }^{3}$ \\ S. Bouchoule ${ }^{3}$ E. Deleporte,${ }^{1}$ C. Voisin, ${ }^{2}$ and J.S. Lauret ${ }^{1, *}$ \\ ${ }^{1}$ Laboratoire Aimé Cotton, CNRS, École Normale Supérieure de Cachan, \\ Université Paris Sud, bât. 505, Campus d'Orsay 91405 Orsay Cedex, France. \\ ${ }^{2}$ Laboratoire Pierre Aigrain, École Normale Supérieure, UPMC, \\ Université Paris Diderot, CNRS (UMR 8551), 75005 Paris, France \\ ${ }^{3}$ Laboratoire Photonique et Nanostructures, CNRS, Route de Nozay, 91460 Marcoussis, France
}

(Dated: March 18, 2013)

\begin{abstract}
We report on the realization of monolithic optical microcavities using a single wall carbon nanotubes doped polymer as active material. Thanks to the control of the polymer thickness, a fine control of the cavity mode energy is achieved, which allows to tune it in exact resonance with a specific chiral species emission line. The quality factor of the filled cavity mode $(Q=40)$ allows to selectively extract the luminescence of the $(7,5)$ chiral species. Finally, angle resolved experiments show the tunability of the emission energy within a $150 \mathrm{meV}$ range.
\end{abstract}

Single Wall Carbon Nanotubes (SWNTs) have attracted much attention for fundamental studies due to their truly one-dimensional geometry and subsequent properties. In particular, their original optical properties make them promising for several optoelectronic applications [1]. The semi-conducting species have a bandgap in the near infrared which can be tuned to optical telecommunication wavelengths by choosing their diameter appropriately [2-4]. One of the most advanced applications regards saturable absorbers for near infrared passive mode-locked pulsed lasers [5], which exploit their huge optical non-linearities [6] together with their fast relaxation rates [7]. In addition, many other high performance optoelectronic devices have been demonstrated such as biological and gas sensors, photo detectors or nano-emitters [8-11].

Recent studies point an important property towards applications as nano-emitters : the photons emitted by a single nanotube show strong anti-bunching, meaning that the photons are emitted one at a time, which is an essential feature for single photon sources [12-14]. A common way to enhance the properties of light emitters is to place them in resonance with the optical mode of a cavity. For instance, this technique can be used to enhance the collection of the emitted photons due to the redirection of light into the mode of the cavity, leading to a very narrow emission cone after the output coupler. Another effect is the acceleration of the radiative recombination rate due to the Purcell effect which allows to increase the brightness of the source. Despite the technological importance of this technique for the use of nanotubes as near-infrared emitters, only a couple of papers have reported on the properties of nanotubes inserted inside an optical cavity, either for nanotubes randomly deposited at the surface of a photonic crystal [15] or in a metal-metal cavity [16] or in a composite micro-cavity [17]. However, the matching of the cavity mode with the nanotube emission is either randomly or approximately achieved due to the technological processes used in these studies. Thus, for practical applications the design of a micro-cavity such that its optical mode is in resonance with the emission of a specific chiral species $(n, m)$ remains an important challenge.

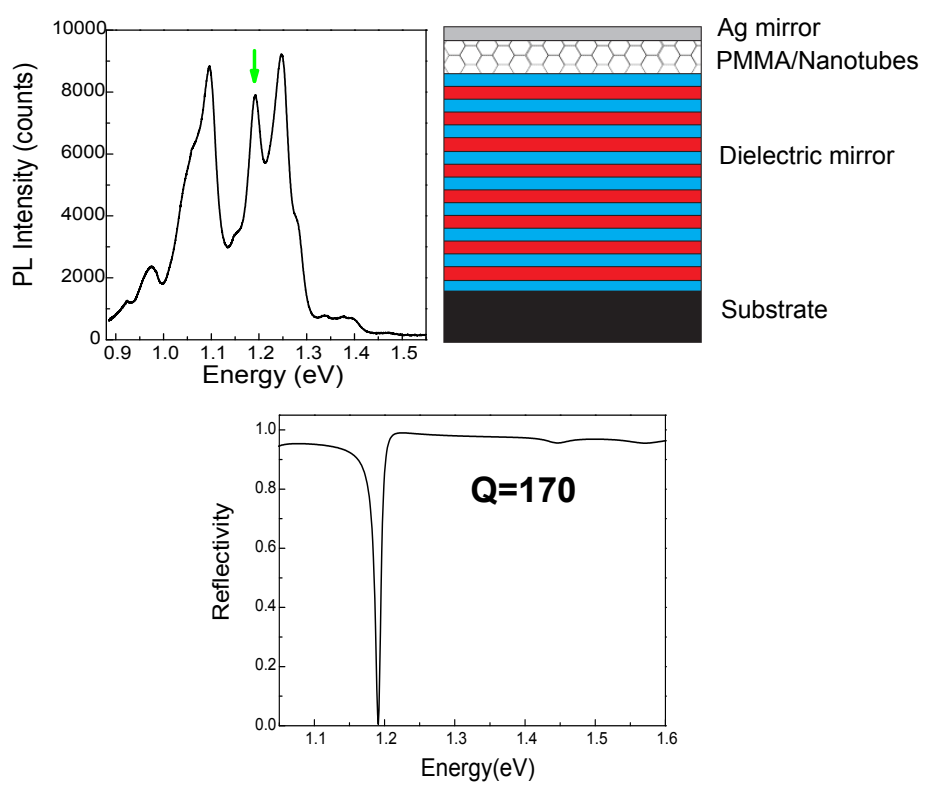

FIG. 1: Photoluminescence spectrum of a PMMA/nanotubes suspension in anisole excited at $2.331 \mathrm{eV}$ (left). Sketch of a monolithic optical microcavity (right). Simulation of the reflectivity spectrum of the cavity for a PMMA/nanotubes layer thickness of $340 \mathrm{~nm}$ (bottom).

In this paper, we report on the realization of monolithic optical microcavities with single wall carbon nanotubes as active material. An exact tuning of the optical mode of the cavity with the $(7,5)$ nanotube emission is achieved through a precise control of the polymer thickness. A quality factor of $Q=40$ for the filled cavity is achieved which corresponds to the selection of the emission of this sole chiral species. Furthermore, angle 
resolved experiments showed the tunability of the emission energy within the inhomogeneous linewidth of that species.

The nanotubes used in this study are synthesized by the CoMoCAT process [18]. The mean diameter of these tubes is about $0.8 \mathrm{~nm}$. The nanotube suspensions are prepared by adding raw nanotubes at $0.15 \mathrm{mg} \cdot \mathrm{mL}^{-1}$ in a solution of PMMA in anisole (10\% wt). The mixture is then sonicated for 2 hours with an ultrasonic tip in order to disperse the nanotubes in the PMMA solution. The reflectivity measurements are performed in a parallel beam configuration with a Xenon lamp as excitation source. The spot size is of the order of $1 \mathrm{~mm}$. The reflected light is dispersed in a monochromator and detected with a $\mathrm{Si}$ photodiode. A laser diode at $2.331 \mathrm{eV}(532 \mathrm{~nm})$ is used as excitation source in the photoluminescence experiments. The spot size on the sample is of the order of $200 \mu \mathrm{m}$, and the power density is $\sim 25 \mathrm{~W} / \mathrm{cm}^{2}$. The emission is collected with a $75 \mathrm{~mm}$ focal length fused silica lens in a 2.1 steradian solid angle (N.A. 0.32). The emission is analyzed through a spectrometer and detected by an InGaAs diodes array (OMA V, Ropers scientific).

Figure 1 displays the photoluminescence spectrum of the PMMA/nanotubes suspension excited at $2.331 \mathrm{eV}$. This excitation energy allows non resonant excitation of the sample. One can observe several lines corresponding to the emission of the lowest exciton $\left(S_{11}\right)$ of each chiral species. Up to 8 different chiral species contribute to the light emission of the sample. The signal level is comparable to the one obtained with regular aqueous micellar suspensions [19] showing the high separation degree obtained with this method in PMMA/anisole solutions. The green arrow in Figure 1 indicates the emission of the $(7,5)$ nanotubes (at $1.192 \mathrm{eV}$ ) on which the following of this study is focused.

The sketch of the $3 \lambda / 4$ cavity is displayed in Figure 1. The PMMA/nanotubes suspension is deposited by spincoating on a $\mathrm{Ta}_{2} \mathrm{O}_{5} / \mathrm{SiO}_{2}$ dielectric mirror (7.5 pairs) and the cavity is then closed with a $30 \mathrm{~nm}$ silver mirror deposited by thermal evaporation. The metal thickness is chosen to optimize the depth of the mode in the reflectivity spectra. The stop-band of the dielectric mirror extends from $1.051 \mathrm{eV}$ to $1.434 \mathrm{eV}$ and is centered at $1.24 \mathrm{eV}$ at normal incidence. The important parameter to control in order to tune the cavity mode in resonance with the desired $(n, m)$ species is the thickness of the PMMA/nanotubes layer. In order to select the $(7,5)$ nanotubes line, the reflectivity spectrum of the cavity has been simulated by the transfer matrix method (bottom of figure 1). The desired thickness of the PMMA/nanotube layer extracted from the simulations is $340 \mathrm{~nm}$. Experimentally, the thickness is controlled by choosing the PMMA concentration and the spin coating parameters. After the deposition, an annealing of 10 minutes at $95^{\circ} \mathrm{C}$ is performed. Profilometer measurements confirmed a thickness of $340 \mathrm{~nm}$. Topography measurements along a

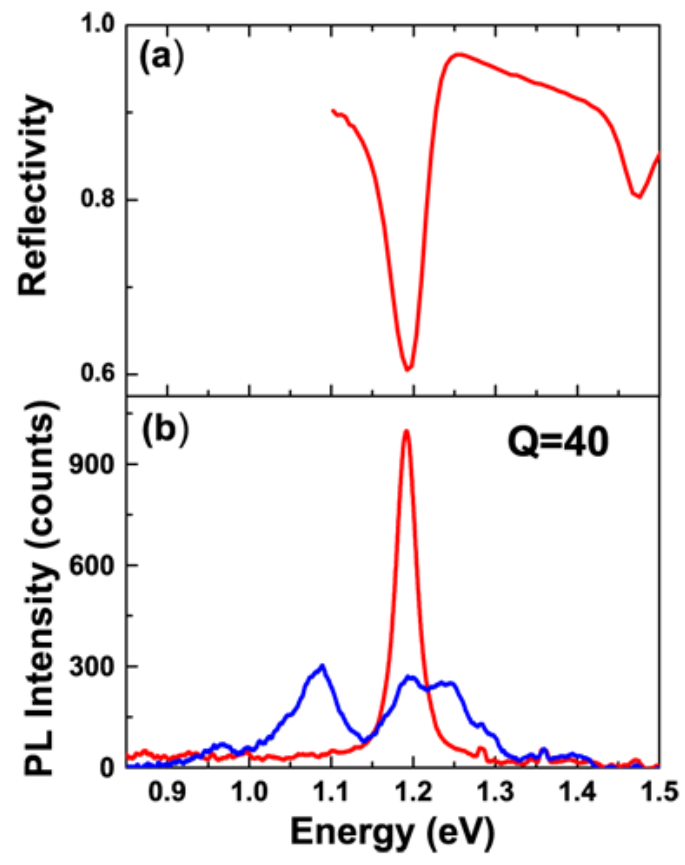

FIG. 2: a) Reflectivity spectrum of the cavity (layer thickness $\sim 340 \mathrm{~nm})$. b) Photoluminescence spectrum of the PMMA/nanotubes layer (blue) and of the cavity excited at $2.331 \mathrm{eV}$ and detected at normal incidence (red).

$1 \mathrm{~mm}$ line show thickness fluctuations of $\pm 20 \mathrm{~nm}$.

Figure 2a) shows the experimental reflectivity spectrum of the cavity containing the PMMA/nanotubes layer. The spectrum displays a hole which corresponds to a cavity mode centered at $1.192 \mathrm{eV}$, that is exactly what was expected from the simulations. The experimental $Q$ factor is estimated to $\sim 30(\Delta \mathrm{E} \sim 39 \mathrm{meV})$, which is quite low in comparison with the theoretical one (170). Let us first consider the role of the losses due to absorption in the PMMA/nanotubes layer in the broadening of the mode. In order to test this contribution, losses were included in the simulations by using a complex effective refraction index for the PMMA/nanotubes layer. The imaginary part was deduced from an optical absorption spectrum of a PMMA/nanotubes layer fitted with a Lorentzian model. The induced broadening $(\sim 200 \mu \mathrm{eV})$ is clearly negligible in comparison with the experimental broadening.

The thickness fluctuations of the layer constitute another source of (inhomogeneous) broadening. The reflectivity measurements were performed with an excitation spot of about $1 \mathrm{~mm}$. At this scale, the reflectivity spectrum results from an average over many cavity domains 
with different thicknesses. We calculated that a variation of $20 \mathrm{~nm}$ of the thickness, which is the order of magnitude of the measured layer roughness, leads to $30 \mathrm{meV}$ shift of the mode. This value is also of the order the broadening. Therefore, we attribute the experimental broadening of the mode mainly to the thickness fluctuations of the PMMA/nanotubes layer.

Figure 2b) shows the photoluminescence spectra of the PMMA/nanotubes layer before closing the cavity (blue curve) and of the cavity (red curve). As in solution, the PL spectrum of the layer consists of several lines. A red shift of $\sim 1 \mathrm{meV}$ and a broadening of $\sim 10 \mathrm{meV}$ of the $(7,5)$ line is observed in comparison with the suspension. These can be due both to changes in the local dielectric environment of the nanotubes and to stress effects induced by the solid matrix [20]. On the contrary, the PL spectrum of the cavity shows only one line centered at $1.192 \mathrm{eV}$ which corresponds to the emission energy of the $(7,5)$ nanotubes and to the energy of the cavity mode. The PL intensity of the $(7,5)$ nanotubes is enhanced by a factor $\sim 3$ in the cavity configuration in comparison with the uncapped layer, which is the sign of the efficient redirection of the emission into the cavity mode. Moreover, the linewidth is about $30 \mathrm{meV}$ which represents a narrowing of about $50 \%$ in comparison with the $(7,5)$ nanotubes emission in the layer $(\Delta E \sim 60 \mathrm{meV})$. The $Q$ factor deduced from the PL measurements is $\sim 40$ which is slightly higher than the one measured in the reflectivity experiments. Actually, in the PL measurements, the spot size is $\sim 200 \mu \mathrm{m}$ whereas it is $\sim 1 \mathrm{~mm}$ in the reflectivity experiments. Therefore, the PL measurements must be a little less sensitive to the thickness fluctuations.

Finally, in order to test the accuracy and the reproducibility of the process, a detuned cavity was build. The mode of the cavity was tuned on the red energy side of the $(7,5)$ emission line at normal incidence. The reflectivity spectra of this cavity as a function of the incident angle is shown in figure $3 \mathrm{a}$ ). The mode of the cavity $(Q \sim 25)$ varies from $1.138 \mathrm{eV}$ to $1.214 \mathrm{eV}$ between $20^{\circ}$ and $40^{\circ}$ covering all the energy range of the $(7,5)$ emission line. The corresponding PL measurements are displayed on figure $3 \mathrm{~b}$ ). Each line is centered at the energy of the cavity mode at the corresponding angle. This shows that we were able to tune the cavity emission across all the $(7,5)$ emission line.

In summary, we built monolithic optical microcavities with single wall carbon nanotubes as active material. In order to demonstrate the efficiency of the process reported here, the mode of the cavity was matched to the $(7,5)$ nanotubes emission. The emission of these nanotubes was coupled to the optical mode of the cavity leading to a quite narrow $(30 \mathrm{meV})$ emission line. The possibility to tune a cavity with the emission of a given class of chirality $(\mathrm{n}, \mathrm{m})$ opens avenues for the fabrication of new carbon nanotubes based light sources.

The authors thank Guillaume Cassabois for fruitful

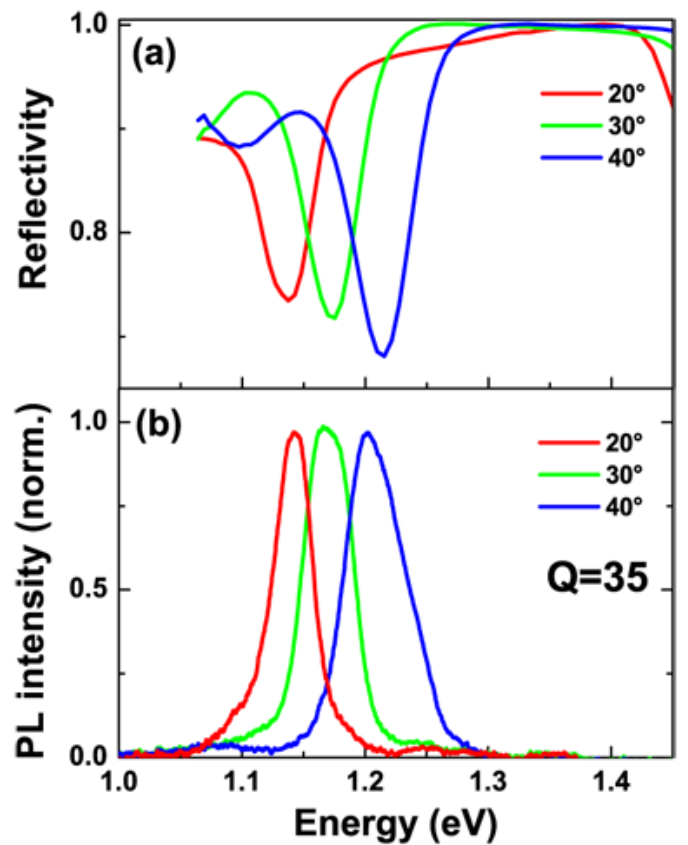

FIG. 3: a) Reflectivity spectra of the detuned cavity for three incident angles; b) Photoluminescence spectra of the detuned cavity, excited at $2.331 \mathrm{eV}$ for three incident angles.

discussions. This work was supported by the GDRI "GNT: Graphene and nanotubes", and ANR grant "TRANCHANT".

* Electronic address: jean-sebastien.lauret@lac. $\mathrm{u}$-psud.fr

[1] P. Avouris, M. Freitag and V. Perebeinos, Nature Photonics 2, 341 (2008).

[2] S.M. Bachilo, M.S. Strano, C. Kittrell, R.H. Hauge, R.E. Smalley, and R.B. Weisman, Science 298, 2361 (2002).

[3] M.J. O'Connel, S.M. Bachilo, C.B. Huffman, V.C. Moore, M.S. Strano, E.H. Haroz, K.L. Rialon, P.J. Boul, W.H. Noon, C. Kittrell, J. Ma, R.H. Hauge, R.B. Weisman, and R.E. Smalley, 297, 593 (2002).

[4] J.S. Lauret, C. Voisin,, G. Cassabois, P. Roussignol, C. Delalande, A. Filoramo, L. Capes, E. Valentin, O. Jost, Physica E 21, 1057 (2004).

[5] S. J. Beecher, R. R. Thomson, N. D. Psaila, Z. Sun, T. Hasan, A. G. Rozhin, A. C. Ferrari, and A. K. Kar, Appl. Phys. Lett. 97, 111114 (2010).

[6] J-S. Lauret, C. Voisin, G. Cassabois, J. Tignon, C. Delalande, and Ph. Roussignol, App. Phys. Lett. 85, 3572 (2004). 
[7] J.S. Lauret, C. Voisin, G. Cassabois, C. Delalande, Ph. Roussignol, O. Jost, and L. Capes, Phys. Rev. Lett. 90, 057404 (2003).

[8] J.H. Kim, D.A. Heller, H. Jin, P.W. Barone, C. Song, J. Zhang, L.J. Trudel, G.N. Wogan, S.R. Tannenbaum, M.S. Strano, Nature Chemistry 1, 473 (2009).

[9] Y. Battie, O. Ducloux, P. Thobois, N. Dorval, J.S. Lauret, B. Attal-Trtout, A. Loiseau, Carbon 49, 3544 (2011).

[10] M. Freitag, Y. Martin, J. A. Misewich, R. Martel, and Ph. Avouris, Nano Letters 3, 1067 (2003).

[11] Michael S. Arnold, Jeramy D. Zimmerman, Christopher K. Renshaw, Xin Xu, Richard R. Lunt, Christine M. Austin, Stephen R. Forrest, Nano letters 9, 3354 (2009).

[12] A. Högele, C. Galland, M. Winger and A. Imamog̃lu, Phys. Rev. Lett. 100, 217401 (2008).

[13] William Walden-Newman, Ibrahim Sarpkaya, and Stefan Strauf, Nano Letters 12, 19341 (2012).

[14] Matthias S. Hofmann, Jan T. Glückert, Alexander Högele, arXiv:1209.3429 , (2012).
[15] R. Watahiki, T. Shimada, P. Zhao, S. Chiashi, S. Iwamoto, Y. Arakawa, S. Maruyama, and Y. K. Kato, Appl.Phys. Lett. 101, 141124 (2012).

[16] F. Xia, M. Steiner, Y.M. Lin and P. Avouris, Nature nano 3, 609 (2008).

[17] E. Gaufres, N. Izard, X. Le Roux, S. Kazaoui, D. MarrisMorini, E. Cassan, and L.Vivien, Optic Express 18, 5740 (2010).

[18] G. Lolli, L. Zhang, L. Balzano, N. Sakulchaicharoen, Y. Tan, and D.E. Resasco, J. Phys. Chem. B 110, 2108 (2006).

[19] C. Roquelet, J.S. Lauret, V. Alain-Rizzo, C. Voisin, R. Fleurier, M. Delarue, D. Garrot, A. Loiseau, Ph. Roussignol, J. A. Delaire, and E. Deleporte, ChemPhysChem 11, 1667 (2010).

[20] S. Berger, F. Iglesias, P. Bonnet, C. Voisin, G. Cassabois, J.-S. Lauret, C. Delalande, and P. Roussignol, J. Appl. Phys. 105, 094323 (2009). 